\title{
Pressions de sélection exercées par le noyau ou le cytoplasme de l'hôte sur l'agressivité d'un parasite nécrotrophe du blé: Septoria nodorum Berk.
}

\author{
F. Rapilly ${ }^{1}$, H. Richard ${ }^{1}$, M. Skajennikoff ${ }^{1}$, Y. Cauderon ${ }^{2}$ et J. Roussel ${ }^{2}$ \\ 1/NRA. station de pathologie végétale, route de Saint Cyr, 78026 Versailles; \\ 2INRA. station de génétique et d'amélioration des plantes, route de Saint Cyr, 78026 Versailles, France
}

(reçu le 26 octobre 1988, accepté le 17 avril 1989)

\begin{abstract}
Résumé - Les pressions de sélection exercées par des éléments de la résistance partielle sur l'agressivité d'une souche de Septoria nodorum, après passages sur des hôtes différents par leurs génomes nucléaires et (ou) cytoplasmiques, permettent l'émergence de souches d'agressivité très variée, confirmant qu'un isolement de $S$. nodorum correspond à une population de génotypes. L'identification, par une série monosomique de blé, des chromosomes de l'hôte impliqués dans l'expression de cette agressivité, permet de constater que les génomes nucléaire et (ou) cytoplasmique exercent des pressions de sélection différentes.

Pour les souches contre-sélectionnées, on peut mettre en évidence des interactions avec les chromosomes de l'hôte. Ces interactions sont très élevées au niveau de la résistance foliaire [durée d'incubation (LPI) et vitesse d'extension des nécroses $\left(P_{\max }\right)$ ], mais sont faibles au niveau de l'expression de la maladie sur les épis (IE). Une certaine spécificité de ces souches vis-à-vis de leur hôte de passage apparaît. Mais il ne semble pas que les rangs de classement de lignées de blé soient changées, confirmant le caractère horizontal de la résistance foliaire. La reconnaissance de souches d'agressivité différentes doit se faire sur les feuilles et non sur les épis. Des indices de similitude peuvent être calculés entre les souches contre-sélectionnées, et les paramètres de résistance pris en compte. Ces indices confirment l'indépendance des résistances foliaires par rapport à celle de l'épi. Ils montrent aussi que ce sont les souches passées sur les cytoplasmes de Triticum timopheevi et de Secale cereale qui sont les plus éloignées l'une de l'autre.
\end{abstract}

résistance horizontale - pression de sélection - noyau - cytoplasme - monosomique - blé - Triticale - Septoria nodorum

Summary - Studies of selection pressures exerted by host cytoplasm or nucleus on the aggressiveness of the necrotrophic fungus Septoria nodorum. This work demonstrates variations in aggressiveness induced by nucleic or cytoplasmic selection pressures exerted on a necrotrophic fungus.

Various selection pressures related to the nucleic or cytoplasmic genomes of isogenic alloplasmic lines of Triticale were applied on S. nodorum strain 6/84. They corresponded to one latent period on leaves and one incubation period on heads, two sequences of general resistante to glume blotch.

The aggressiveness of new strains named, 6/T533-8, 6/T762, 6/T832 (Table I), and of the original strain (6/84) were read on a complete wheat monosomic series inoculated at bost and heading stage. The reponses of the $F_{2}$ monosomics were compared with the $F_{2}$ disomics for the following character: two partial resistance components (incubation time or $L P I$, rate of necrosis extension or $P_{\max }$ ), one tolerance component (disease intensity on heads or IE).

Table II shows the results for the 4 strains. After selection pressure the new strains differed both between themselves and from the original strain for LPI (Tables III and IV), for $P_{\max }$ (Tables VII and VIII) and for IE (TablesXI and XII). The effects induced by host nucleus differed from those of cytoplasm and also of nucleo-cytoplasmic interactions. The 3 types of cytoplasm (T. aestivum, T. timopheevi and Secale cereale) gave few common responses for monosomics, irrespective of the strain. For $L P I$, only $F_{2} 6 D$ displayed the same susceptibility reaction for the 4 strains (Table IV). Two by two comparisons of strains showed differential interactions with monosomics (Table VI). For the incubation time a very low coefficient of relationship was observed for the strains obtained on T. timopheevi or Secale cereale cytoplasm.

For $P_{\max }$, no monosomics displayed the same resistance or susceptibility reactions for all the strains (Table VIII). The strain derived from Secale cereale cytoplasm was significantly different from that derived from T. timopheevi cytoplasm. Important differential interactions (Table IX) were calculated for wheat genomes and for homologous groups. Interactions due to nuclear effects differed from those produced by cytoplasmics effects (Table X). As with LPI the 
coefficient of relationship between 6/T832 and 6/T762 was very low; it was higher between 6/84 and 6/T533-8, which allowed comparison of nucleus selection pressure.

For IE few differences were observed between the 3 strains obtained after cytoplasmic effects; but these strains greatly differed from the original strain (Table XII). Differential interactions were also calculated (Table XIII); these were less important than for $P_{\max }$ and, in many cases, could not be detected (Table XIV). Strains 6/T533-8 and 6/T832, and $6 / T 762$ and $6 / 7832$, were the pairs with the best relationship coefficient (Table XVIII), in contradiction to the behaviours of these strains for the leaf parameters.

For all the strains the relations between $L P I$ and $P_{\max }$ occurred again. However, the slope of the linear regression for $6 / T 832$ significantly differed from the others (Table XV). No correlations were found between LPI and IE or between $P_{\max }$ and IE. Few monosomics displayed the same responses to the 3 parameters (Table XVII) or when these were involved in common interactions (Table XVI).

All the strains acquired some specificity with a new level of pathogenicity toward their parasitic hosts; for example, the Secale cereale cytoplasm gave a strain which is pathogenic for Secale cereale as a host (Table XIX). The ranking of wheat varietes appeared to be the same as that obtained with the original strain if the criterium used is the epidemic progression of the disease (parameter of horizontal resistance). But these rankings differed between strains if the criterium used is the loss of thousand Kernel weight (parameter of tolerance).

These results bring up many questions on the specificity of isolates in the case of partial resistance, and also on the necessity of taking into account the effects of cytoplasmic genomes on the variability of parasitic fungi.

The origin of these variations of aggressiveness, wether acquired or revealed, is discussed. Practical considerations are made for breeding wheat resistant to Septoria nodorum. It is also confirmed that the leaf aspects of this disease are very different from, but perhaps complementary to, head resistance or tolerance.

horizontal resistance - selection pressure - nucleus - cytoplasm - monosomic - wheat - Triticale - Septoria nodorum

\section{INTRODUCTION}

Des publications précédentes (Auriau et al., 1988; Rapilly et al., 1988a et b) nous ont montré que des critères de résistance foliaire partielle du blé à Septoria nodorum et ceux dits de tolérance relèvent de 2 systèmes polygéniques indépendants l'un de l'autre. Nous avons aussi constaté que les chromosomes de l'hôte, impli-qués dans le contrôle d'un même ensemble de caractères, variaient avec l'origine de la souche, sans pouvoir préciser si ces variations reflétaient le fait qu'un même symptôme pouvait être contrôlé par des parts différentes du génotype du parasite. D'autres travaux (Osbourn 1985, Skajennikoff et Rapilly, 1989) démontrent que les spores d'une même pycnide donnent naissance à une population dont les éléments constitutifs ont des pouvoirs pathogènes différents.

La relation de résistance, gène pour gène, mise en évidence par Flor (1955) se traduit par une pression de sélection, favorisant la prédominance d'éléments qui, au sein de la population parasitaire, portent les gènes nécessaires au contournement de ces résistances, souvent monogéniques et qui sont qualifiées de non durables. La résistance partielle polygénique, dite aussi horizontale est, elle, qualifiée de durable. Mais la démonstration reste à faire que ce type de résistance n'exerce pas, sur le parasite, de pressions de sélection favorisant l'émergence de nouvelles agressivités. Beye \& Lafay (1988) ont montré que la résistance générale de lignées de tomate à Verticillium dahliae Kleb. pouvait favoriser les éléments du parasite les plus aptes à se multiplier sur un lignée donnée. Nous-même (Skajennikoff \& Rapilly, 1985) avons observé, pour $S$. nodorum, qu'un changement d'hôte induisait un changement d'agressivité de la souche utilisée, dont les éléments les plus aptes à cet hôte étaient favorisés.

Par l'identification exacte des chromosomes de l'hôte, que permet une série monosomique, il est possible de réaliser des analyses fines pour juger et quantifier les variations de structure de l'agressivité d'un isolat de Septoria nodorum, soumis à des pressions de sélection par passage sur des hôtes différents par leurs génomes nucléaires et (ou) cytoplasmiques. Les résultats présentés ici apportent des éléments de réponse, d'une part, à la stabilité des composants de la résistance partielle et, d'autre part, à la variabilité de l'agressivité de $S$. nodorum, parasite nécrotrophe.

\section{MATÉRIEL ET MÉTHODES}

\section{Matériel végétal}

II concerne les hôtes de passage, sur lesquels la même souche de $S$. nodorum est inoculée, et la série monosomique, sur laquelle sont déterminés les chromosomes de l'hôte qui répondent à l'agressivité du parasite.

\section{Hôtes de passage}

Ils correspondent à une série de Triticale hexaploïde qui est isogénique et alloplasmique, dont l'origine est décrite par Cauderon et al. (1985). Cette série a déjà 
été utilisée dans nos études (Skajennikoff \& Rapilly $1985,1989)$ pour juger de la variabilité du parasite. Leurs caractéristiques (Tableau I) permettent, par référence au blé "Etoile de Choisy", d'exercer sur la population du parasite diverses pressions de sélection. Rappelons que le génotype nucléaire du blé a la composition $A, B, D$, alors que celui des Triticale hexaploïdes est $A, B, R$.

\section{Hôtes de lecture}

La lecture de l'agressivité des souches du parasite est réalisée sur la série des familles $F_{2}$ issues des $F_{1}$ monosomiques du croisement entre la lignée $L 22$ et "Courtôt" monosomique. Comme dans nos études précédentes (Rapilly 1988 a et b), la $F_{2}$ disomique sert de terme de comparaison. Seul le monosomique 7D est absent, les semences correspondantes n'ayant pas levé.

\section{Souches de S. nodorum utilisées}

Toutes les souches utilisées ont pour origine la population $6 / 84$, isolée de grains de blé "Etoile de Choisy". Cette souche a permis, par inoculation plurispore, de contaminer des limbes foliaires, maintenus en survie, des 3 Triticale. Après sporulation, des souches nouvelles sont réisolées et prennent la dénomination de $6 / T$ 533-8, 6/T 762 et 6/T 832; ce sont aussi des populations. Elles sont alors utilisées pour contaminer les épis correspondant aux hôtes de passage. C'est à partir des grains contaminés, en 1984, que ces souches sont de nouveau isolées, en 1988, pour réaliser cette étude.

Chaque souche a donc accompli, sur feuille, une période de latence (de la contamination à la sporulation) et, sur épi, l'envahissement des glumes et des enveloppes du grain, soit une période d'incubation. Les pressions de sélection correspondent donc à celles qu'exercent des éléments de la résistance générale. Le très faible nombre de cycles accomplis sur l'hôte de passage (un et demi) permet de penser que les comparaisons effectuées révèlent l'effet de "filtre" des hôtes de passage sur une population initiale hétérogène, plutôt que des remaniements chromosomiques.

\section{Dispositif utilisé}

Il est identique à celui décrit dans nos travaux précédents (Auriau et al., 1988; Rapilly et al., 1988a). Chaque parcelle élémentaire correspond à 1 ligne de 20 plantes individualisées, issues de la même $F_{1}$ monosomique. Une répétition comprend les 20 monosomiques, plus trois lignes de $F_{2}$ disomiques et 3 lignes d'une variété très sensible, qui permettent de juger, précocement, de la réussite des contaminations. Ces lignes sont réparties au hasard. Une répétition est ainsi constituée, par souche étudiée. Les contaminations sont réalisées aux stades du gonflement et de la fin de l'épiaison. II n'y a pas de témoin sain.

\section{Notations et interprétations réalisées}

\section{Notations}

Plusieurs notations, espacées de 3 à $4 \mathrm{j}$, réalisées plante à plante, sont effectuées; elles portent sur les critères utilisés dans nos autres études (Rapilly et al., 1988a) à savoir:

- la durée d'incubation (LPI)

- l'intensité des nécroses foliaires, qui reflète la vitesse d'extension des nécroses foliaires $\left(P_{\max }\right)$, car il n'y a pas eu de recontamination pendant la période de notation.

- l'intensité des symptômes sur épis (IE)

Tableau I. Caractéristiques des hôtes de passage utilisés, dénomination des souches, pressions de sélection exercées et comparaisons effectuées.

\begin{tabular}{|c|c|c|c|}
\hline $\begin{array}{c}\text { Hôtes de passage et } \\
\text { caractéristiques }\end{array}$ & $\begin{array}{l}\text { Dénomination } \\
\text { des souches }\end{array}$ & $\begin{array}{l}\text { Comparaisons } \\
\text { effectuées }\end{array}$ & Pressions de sélection exercées \\
\hline
\end{tabular}

- blé : cv. Etoile de Choisy

cytoplasme T. aestivum

- Triticale : T533-8 (génotype

T533-8, cytoplasme T. aestivum)

- Triticale : T762 (génotype

T533-8, cytoplasme T. timopheevi)

- Triticale : T832 (génotype

T533-8, cytoplasme Secale cereale)
$6 / 84$

\begin{tabular}{|c|c|c|}
\hline $6 / T 533-8$ & $6 / 84$ et $6 / T 533-8$ & génome nucléaire de T 533-8 \\
\hline $6 / 7762$ & $6 /$ T533-8 et $6 /$ T762 & $\begin{array}{l}\text { cytoplasme de } T \text {. timopheevi par } \\
\text { rapport au cytoplasme de } T \text {. aestivum }\end{array}$ \\
\hline 6/Т832 & $6 /$ T533-8 et $6 /$ T832 & $\begin{array}{l}\text { cytoplasme de Secale cereale par } \\
\text { rapport au cytoplasme de T. aestivum }\end{array}$ \\
\hline & $6 /$ T762 et $6 /$ T832 & $\begin{array}{l}\text { cytoplasme de Secale cereale par } \\
\text { rapport au cytoplasme de T. timopheevi) }\end{array}$ \\
\hline & $6 / 84$ et $6 / T 762$ & $\begin{array}{l}\text { génotype nucléaire de T533-8 et } \\
\text { cytoplasme de } T \text {. timopheevi) }\end{array}$ \\
\hline & $6 / 84$ et $6 / T 832$ & $\begin{array}{l}\text { génotype nucléaire de T533-8 et } \\
\text { cytoplasme de Secale cereale) }\end{array}$ \\
\hline
\end{tabular}




\section{Interprétations}

Les analyses de variance sont réalisées soit à partir des notes moyennes calculées pour chaque $F_{2}$ monosomique, en comparaison avec la $F_{2}$ disomique, soit à partir des notations, plante à plante, pour mettre en évidence les interactions, qui sont calculées au seuil de $5 \%$. Ces dernières sont identifiées par des représentations graphiques, non présentées. Pour la LPI, le critère utilisé est l'aire sous la courbe du nombre de plantes, en fin d'incubation, en fonction du temps: plus l'aire est réduite, plus la résistance est grande et inversement. Ce critère ne permet pas d'utiliser les notations individuelles; pour rechercher les interactions, un calcul de corrélation de rangs est alors réalisé. En outre, nous avons calculé, pour les $F_{2}$ disomiques et à partir des notations individuelles, l'amplitude de variations au seuil de $10 \%$ de la LPI, pour chaque souche.

\section{Indices de similitudes}

La connaissance du nombre de chromosomes impliqués dans les interactions, par rapport au nombre total de chromosomes étudiés (ici 20), permet de calculer un indice de similitude pour chacune des comparaisons effectuées entre souches et pour chaque paramètre pris en compte. Cet indice, dont la valeur est comprise entre 0 et 1 , est calculé de la façon suivante: indice $=1-\frac{\text { Nombre de chromosomes impliqués dans l'interaction }}{\text { Nombre de chromosomes étudiés (ici 20) }}$

Si indice $=0$, il n'existe aucune similitude entre les souches comparées; si indice $=1,2$ souches sont, pour le paramètre étudié, similaires.

\section{RÉSULTATS}

Les résultats sont présentés souche par souche, pour chaque paramètre de résistance étudié, puis les interactions entre les souches sont recherchées et les indices de similitudes calculés. Le Tableau II présente les principaux résultats obtenus, ils sont exprimés en \% des disomiques correspondants.

\section{Durée d'incubation (LPI)}

\section{Comparaisons globales}

Globalement, il n'existerait pas de différences entre les monosomiques, mais les souches se

Tableau II. Valeurs relatives de chaque ligne $F_{2}$ monosomique pour la durée d'incubation (LPI), la vitesse d'extension des nécroses foliaires $\left(P_{\max }\right)$ et l'intensité des symptômes sur épis (IE) exprimées en \% de la combinaison $F_{2}$ disomique, souche correspondante.

\begin{tabular}{|c|c|c|c|c|c|c|c|c|c|c|c|c|}
\hline \multirow{2}{*}{$\begin{array}{c}F_{2} \\
\text { Monosomique }\end{array}$} & \multicolumn{3}{|c|}{ Souche $6 / 84$} & \multicolumn{3}{|c|}{ Souche $6 / T 533-8$} & \multicolumn{3}{|c|}{ Souche $6 / T 762$} & \multicolumn{3}{|c|}{ Souche $6 / T 832$} \\
\hline & $L P(1)$ & $P_{\max }^{(2)}$ & $I E(2)$ & $L P\left({ }^{(1)}\right.$ & $P_{\max }^{(2)}$ & $I E(2)$ & $L P(1)$ & $P_{\max }^{(2)}$ & $I E$ & $L P I$ & $P_{\max }$ & $I E$ \\
\hline $1 \mathrm{~A}$ & 106,8 & 98 & 121,3 & 109,2 & 104,5 & 100 & 97,5 & 92,2 & $138,8^{*}$ & $152,2^{*}$ & $118,6^{*}$ & $186,2^{*}$ \\
\hline $2 A$ & $52^{*}$ & $78,7^{\star}$ & $63,8^{*}$ & $112,8^{*}$ & $109,9^{*}$ & 100 & 90,7 & 92,2 & 116,4 & $125,8^{\star}$ & 95,8 & 124,1 \\
\hline $3 \mathrm{~A}$ & 102,9 & 101,2 & 92,5 & $161,7^{*}$ & $123,6^{\star}$ & $200^{*}$ & $79,5^{\star}$ & $81,1^{*}$ & 71,6 & 97,7 & $79,6^{*}$ & $206,9^{*}$ \\
\hline $4 \mathrm{~A}$ & $84,8^{*}$ & 92,9 & $67^{*}$ & $134,9^{*}$ & $113,2^{*}$ & $153,3^{*}$ & $129,9^{*}$ & $123,6^{*}$ & 98,5 & $111,7^{\star}$ & $86,7^{*}$ & 51,7 \\
\hline $5 \mathrm{~A}$ & 99,5 & 105 & $47,9^{\star}$ & 110,5 & 99 & 92,8 & $76,9^{*}$ & $75,6^{\star}$ & 71,6 & 99,8 & $118,7^{\star}$ & 62,1 \\
\hline $6 \mathrm{~A}$ & 108,3 & $87,5^{\star}$ & 73,4 & $136,6^{\star}$ & $89,9^{*}$ & 133,3 & 88,1 & $77,1^{*}$ & 103 & $144,6^{*}$ & $115,8^{*}$ & 51,7 \\
\hline $7 \mathrm{~A}$ & 101,9 & $73,5^{*}$ & 124,5 & 124,7 & 106 & 140 & 97,3 & $81,8^{*}$ & 103 & $155,5^{\star}$ & 92,3 & 82,7 \\
\hline $1 \mathrm{~B}$ & $113,2^{*}$ & 96,3 & $51,1^{*}$ & 87,3 & 98,8 & $186,7^{*}$ & 102,7 & $120^{*}$ & 89,5 & $143,6^{*}$ & 107 & 144,8 \\
\hline $2 \mathrm{~B}$ & 90,5 & $78,8^{*}$ & 83 & $149,5^{*}$ & $120,4^{*}$ & 80 & 90,1 & $114,4^{*}$ & $147,7^{\star}$ & 73,6 & $80,7^{*}$ & 134,5 \\
\hline $3 B$ & 99 & $73,5^{*}$ & $31,9^{*}$ & $118,8^{*}$ & 103,2 & 113,3 & $141,8^{*}$ & $127,3^{*}$ & 98,5 & 102,6 & 97 & 134,5 \\
\hline $4 \mathrm{~B}$ & $127,1^{*}$ & 96,3 & 124,4 & $144,1^{*}$ & $122,2^{*}$ & $153,3^{*}$ & 90,9 & 91 & $58,2^{*}$ & $133,1^{*}$ & $113^{*}$ & $289,6^{*}$ \\
\hline $5 B$ & $127,1^{*}$ & $131,3^{*}$ & 117 & $126,8^{*}$ & $87,6^{*}$ & $180^{*}$ & $84,4^{*}$ & 92,2 & 107,5 & $86,3^{*}$ & $72,4^{*}$ & 103,4 \\
\hline $6 \mathrm{~B}$ & $144,3^{*}$ & $90^{*}$ & $47,9^{*}$ & $144,3^{*}$ & $112,9^{*}$ & 85,7 & $117,5^{\star}$ & 103,8 & $134,3^{*}$ & 97,7 & $81,4^{*}$ & 62,1 \\
\hline $7 \mathrm{~B}$ & 88.2 & 105 & $67^{\star}$ & $144,3^{*}$ & $136,6^{*}$ & 78,6 & 90.1 & 92,2 & 85,1 & 110,3 & 93,2 & 155,1 \\
\hline $1 \mathrm{D}$ & $82,3^{*}$ & $73,5^{*}$ & 108,5 & 108,1 & 97,7 & $173,3^{*}$ & 95,9 & $114,4^{*}$ & 116,4 & $143^{*}$ & $111,5^{\star}$ & 134,5 \\
\hline $2 \mathrm{D}$ & 97,5 & $77^{*}$ & $70,2^{*}$ & $83,5^{\star}$ & 94,4 & $173,3^{*}$ & $78,1^{*}$ & 98,6 & $138,8^{*}$ & 105,4 & 100,2 & $245,7^{*}$ \\
\hline $3 \mathrm{D}$ & 91,3 & $71,8^{\star}$ & 86,2 & $114,7^{*}$ & $85,9^{*}$ & 146,7 & 123,2 & 96 & 76,1 & 103,4 & $89,6^{\star}$ & 165,5 \\
\hline $4 \mathrm{D}$ & 105,5 & $125^{*}$ & 102,1 & 97,8 & 102,4 & 80 & $146,8^{*}$ & $118^{*}$ & $179,1^{*}$ & 94 & $89,7^{\star}$ & 82,7 \\
\hline $5 \mathrm{D}$ & $113,4^{*}$ & 105 & 76,6 & $145,8^{*}$ & $139,3^{*}$ & 128 & 100,4 & $117,6^{*}$ & 89,5 & 86,2 & 101,3 & 134,5 \\
\hline $6 \mathrm{D}$ & $150,1^{*}$ & $124,7^{\star}$ & 111,7 & $173,1^{*}$ & $125,8^{*}$ & 86,7 & $143,4^{*}$ & $134,7^{\star}$ & 89,5 & $156,4^{*}$ & $83,9^{*}$ & 62,1 \\
\hline \multirow[t]{2}{*}{$F_{2}$ Dis } & 100 & 100 & 100 & 100 & 100 & 100 & 100 & 100 & 100 & 100 & 100 & 100 \\
\hline & $=42,57$ & $=29,56$ & $4=29,83$ & $=40,23$ & $=29,67$ & $=15$ & $=44,56$ & $=27,1$ & $=22,33$ & $=43,04$ & $=34,54$ & $=9,68$ \\
\hline
\end{tabular}

$(1)^{*}=$ valeur inférieure ou supérieure à l'amplitude de variation au seuil de $10 \%$ des $F_{2}$ disomiques.

(2) * = différent des disomiques au seuil minimum de $5 \%$ 
distinguent entre elles (Tableau III). Par durée croissante de la L.P.I. elles se classent ainsi:

6/T533-8, 6/T832, $6 / 84$ et $6 / T 762$
(A)
(AB)
(B)
(B)

Le changement de génome nucléaire de l'hôte (comparaison 6/24 et 6/T533-8) permet l'émergence d'une souche qui, pour ce caractère, est plus agressive que la population originelle.

L'action des seuls cytoplasmes permet de classer les souches ainsi:

\section{6/T533-8, 6/T832, 6/T762}
(A)
(AB)
(B)

Le cytoplasme de $T$. timopheevi entraîne une déviation de la population vers une agressivité, pour ce caractère, globalement plus faible que le cytoplasme $T$. aestivum. Celui de $S$. cereale donne une situation intermédiaire.

\section{Comparaisons entre les monosomiques}

L'amplitude de réponse des plantes disomiques prises individuellement, calculée au seuil de $10 \%$, correspond à environ plus ou moins $13 \%$ pour toutes les souches; pour comparer les monosomiques aux disomiques, nous avons retenu cette valeur de $13 \%$. Elle permet de distinguer les monosomiques pour lesquels la LPI est allongée (réponse de résistance) de ceux pour lesquels elle semble réduite (réponse de sensibilité) (Tableau IV). II est remarquable de constater que, vis-à-vis des diverses souches, seul le monosomique $6 \mathrm{D}$ donne toujours la même réponse: une réduction de la $\mathrm{LPI}$, donc un accroissement de la sensibilité. De très nombreuses inversions de réponses peuvent être relevées; c'est le cas des $1 \mathrm{~B}, 2 \mathrm{~A}$ entre les souches $6 / 84$ et $6 / T$ 533-8, des $1 D, 2 A, 4 A$ entre

Tableau III. Analyse de variance, des réponses des monosomiques, exprimées en $\%$ de la $F_{2}$ disomique contaminée par chaque souche de $S$. nodorum, pour la durée d'incubation (LPI). Le critère de jugement est l'aire située sous la courbe de l'évolution dans le temps du \% de plantes atteignant la fin d'incubation.

\begin{tabular}{lcccccc}
\hline Variations & Somme des carrés & ddl & CM & $F$ & \multicolumn{2}{c}{$\begin{array}{c}\text { F théorique } \\
5 \%\end{array}$} \\
& & & & & & \\
& & & & & & \\
\hline & & & & & & \\
totale & 49644,5 & 3 & 2403,25 & 4,641 & 2,78 & 4,16 \\
souches & 7209,74 & 19 & 680,1 & 1,313 & 1,75 & 2,20 \\
$F_{2}$ monosomiques & 12920,28 & 57 & 517,81 & & & \\
erreur & 29514,97 & & &
\end{tabular}

Tableau IV. Chromosomes de l'hôte qui semblent, pour chaque souche, allonger ou réduire la durée d'incubation (L.P.I) ou qui sont équivalents à la $\mathrm{F}_{2}$ disomique.

Souches

Durée d'incubation (LPI)

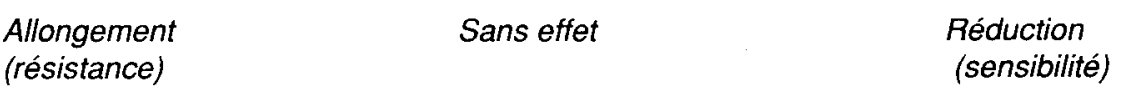

$\begin{array}{llll}6 / 84 & 2 A-4 A & 1 A-3 A-5 A-6 A-7 A & \\ & & 2 B-3 B-7 B & \\ 2 D-3 D-4 D & 1 B-4 B-5 B-6 B \\ & 1 D & & 5 D-6 D \\ 6 / 7533-8 & & 1 A-5 A & 2 A-3 A-4 A-6 A-7 A \\ & 1 B & 1 D-4 D & 2 B-3 B-4 B-5 B-6 B-7 B \\ & 2 D & & 3 D-5 D-6 D \\ 6 / T 762 & 3 A-5 A & 1 A-2 A-6 A-7 A & 4 A \\ & 5 B & 1 B-2 B-4 B-7 B & 3 B-6 B \\ & 2 D & 1 D-5 D & 3 D-4 D-6 D \\ 6 / 7832 & & 3 A-5 A & 1 A-2 A-4 A-6 A-7 A \\ & 2 B-5 B & 3 B-6 B-7 B & 1 B-4 B \\ & & 2 D-3 D-4 D-5 D & 1 D-6 D\end{array}$


$6 / 84$ et $6 / \mathrm{T}$ 832, des $2 \mathrm{~B}, 5 \mathrm{~B}$ entre $6 / \mathrm{T} 533-8$ et $6 / T$ 832, et des chromosomes $3 A, 5 B$ entre $6 / T$ 762 et $6 / \mathrm{T} 533-8$.

Par rapport à l'effet des 3 cytoplasmes, seuls les $4 \mathrm{~A}$ et $6 \mathrm{D}$ donnent une même réponse de sensibilité et aucun monosomique n'est commun pour la résistance. Ces exemples montrent qu'il existe des interactions "monosomique $x$ souche", comme le laisse supposer la valeur élevée du carré moyen de l'erreur (Tableau III).

\section{Intéractions monosomique $\mathrm{x}$ souche}

Celles-ci peuvent être mises en évidence en calculant les coefficients de corrélation de rangs entre les classements des monosomiques pour chaque souche. La valeurs du rs de Spearmann, pour les 6 comparaisons étudiées, montre qu'aucun de ces coefficients n'est significatif (Tableau $V$ ), ce qui traduit des effets différentiels entre les monosomiques et les souches. La représentation graphique, non présentée ici, des résultats du Tableau II permet d'identifier des interactions très élevées (réponse passant de résistant à sensible et inversement) (Tableau VI), ou plus réduites (réponse passant de résistant ou sensible à sans effet et inversement). Ces interactions sont décelées dans toutes les comparaisons effectuées: changements des génomes nucléaire ou cytoplasmique, effets de ces 2 génomes associés. Ce sont les souches $6 /$ T762 et $6 /$ T832 qui apparaissent comme étant les plus éloignées l'une de l'autre, puisqu'elles n'ont que 5 chromosomes sur

Tableau V. Coefficients de corrélation des rangs de Spearmann pour la L.P.I. et les 6 comparaisons effectuées.

\begin{tabular}{|c|c|c|c|c|c|c|}
\hline & \multicolumn{6}{|c|}{ Comparaisons effectuées (1) } \\
\hline & $\begin{array}{c}6 / 84 \text { et } \\
6 / T 533-8\end{array}$ & $\begin{array}{l}6 / T 533-8 \\
\text { et } 6 / T 762\end{array}$ & $\begin{array}{l}6 / T 533-8 \\
\text { et } 6 / T 832\end{array}$ & $\begin{array}{c}6 / T 762 \\
\text { et } 6 / T 832\end{array}$ & $\begin{array}{l}6 / 84 \text { et } \\
6 / T 762\end{array}$ & $\begin{array}{l}6 / 84 \text { et } \\
6 / \text { T832 }\end{array}$ \\
\hline Valeurs de $r s$ & 0,309 & 0,007 & $-0,178$ & 0,158 & 0,191 & 0,04 \\
\hline
\end{tabular}

(1) Pour 18 ddi rs $=0444$ à 5 p. 100.

Tableau VI. Récapitulatif des chromosomes de l'hôte impliqués, pour la durée d'incubation (LPI), dans des interactions avec les souches du parasite.

\section{Chromosomes}

\begin{tabular}{|c|c|c|c|}
\hline $\begin{array}{l}\text { Comparaisons } \\
\text { effectuées }\end{array}$ & $\begin{array}{l}\text { Interactions élevées } \\
\text { (de résistant à sensible } \\
\text { et inversement) }\end{array}$ & $\begin{array}{l}\text { Interactions réduites } \\
\text { (de résistant ou sensible } \\
\text { à sans effet et inversement) }\end{array}$ & $\begin{array}{l}\text { Sans effet } \\
\text { différentiel }\end{array}$ \\
\hline $\begin{array}{l}6 / 84 \text { et } 6 / T 533-8 \\
\text { effet génotype nucléaire de T533-8 }\end{array}$ & $\begin{array}{l}2 A-4 A \\
1 B\end{array}$ & $\begin{array}{l}3 A-6 A-7 A \\
2 B-3 B-7 D \\
1 D-2 D-3 D\end{array}$ & $\begin{array}{l}\frac{1 A-5 A}{4 B-5 B-6 B} \\
\underline{4 D}-5 D-6 D\end{array}$ \\
\hline $\begin{array}{l}6 / T 533-8 \text { et } 6 / T 762 \\
\text { effet cytoplasme } \\
T . \text { aestivum, T. timopheevi }\end{array}$ & $\begin{array}{l}3 A \\
5 B\end{array}$ & $\begin{array}{l}2 A-5 A-6 A-7 A \\
1 B-2 B-4 B-7 B \\
4 D-5 D\end{array}$ & $\begin{array}{l}\frac{1 A-4 A}{3 B}-6 B \\
1 D-2 D-3 D-6 D\end{array}$ \\
\hline $\begin{array}{l}6 / \text { T533-8 et } 6 / \text { T } 832 \\
\text { effet cytoplasme } \\
\text { T. aestivum, Secale cereale }\end{array}$ & 1B-2B-5B & $\begin{array}{l}1 A-3 A \\
3 B-6 B-7 B \\
1 D-2 D-3 D-5 D\end{array}$ & $\begin{array}{l}2 A-4 A-5 A-6 A-7 A \\
4 B \\
4 D-6 D\end{array}$ \\
\hline $\begin{array}{l}6 / T 762 \text { et } 6 / T 832 \\
\text { effet cytoplasme } \\
\text { T. timopheevi, Secale cereale }\end{array}$ & & $\begin{array}{l}1 A-2 A-3 A-5 A-6 A-7 A \\
1 B-2 B-3 A-4 B-6 B \\
1 D-2 D-3 D-4 D\end{array}$ & $\begin{array}{l}4 \mathrm{~A} \\
5 \mathrm{~B}-7 \mathrm{~B} \\
\underline{5 \mathrm{D}-6 \mathrm{D}}\end{array}$ \\
\hline $\begin{array}{l}6 / 84 \text { et } 6 / T 762 \\
\text { effet génotype nucléaire de T533-8 } \\
\text { plus cytoplasme de } T . \text { timopheevi }\end{array}$ & $\begin{array}{l}4 \mathrm{~A} \\
5 \mathrm{~B}\end{array}$ & $\begin{array}{l}2 A-3 A-5 A \\
1 B-3 B-4 B \\
1 D-2 D-3 D-4 D-5 D\end{array}$ & $\frac{1 A}{2 B}-\frac{6 A}{6 D}-\frac{7 A}{7 B}$ \\
\hline $\begin{array}{l}6 / 84 \text { et } 6 / T 832 \\
\text { effet génotype nucléaire de T533-8 } \\
\text { plus cytoplasme de Secale cereale }\end{array}$ & $\begin{array}{l}2 A-4 A \\
5 B \\
1 D\end{array}$ & $\begin{array}{l}1 A-6 A-7 A \\
2 B-6 B \\
5 D\end{array}$ & $\begin{array}{l}\frac{3 A}{1 B}-\frac{5 A}{3 B}-4 B-7 B \\
2 D-3 D-4 D-6 D\end{array}$ \\
\hline
\end{tabular}


20 qui donnent une réponse de même sens, dont le $5 \mathrm{D}$, qui est le seul à donner une réponse de résistance. Les $4 A$ et $6 \mathrm{D}$ réduisent la L.P.I.

\section{VITESSE D'EXTENSION DES NÉCROSES FOLIAIRES ( $P_{\text {MAX) }}$}

\section{Comparaisons globales}

L'analyse de variance faite à partir de la note moyenne de chaque lignée monosomique, exprimée en $\%$ de la $F_{2}$ disomique correspondante, montre que, tous monosomiques confondus, il n'existerait de différences qu'entre les souches (Tableau VII).

Celles-ci, par ordre croissant de $P_{\max }$, se classent ainsi:

$6 / 84, \quad 6 / T 762, \quad 6 / T 533-8, \quad 6 / T 832$
(A)
(A)
(AB)
(B)

Le changement du génome nucléaire de l'hôte permet l'émergence d'une souche 6/T533-8 légè- rement plus agressive que la population originelle. Le cytoplasme de $S$. cereale permet de révéler une souche différente de celle obtenue sur le sytoplasme de T. timopheevi. Le cytpoplasme de $T$. aestivum fournit, lui, une souche intermédiaire entre les deux précédentes.

\section{Comparaisons entre les monosomiques}

Le Tableau VIII donne, pour chaque souche, le positionnement des monosomiques par rapport aux disomiques. II montre l'émergence à partir de la population $6 / 84$, de souches très différentes en fonction des pressions de sélection qu'elles ont subies. II n'existe pas de monosomique donnant une même réponse aux 4 souches, y compris parmi ceux qui sont équivalents aux $F_{2}$ disomiques.

Le changement du génome nucléaire de l'hôte de passage permet de révéler une souche $(6 / T$ 533-8) qui n'a que 6 monosomiques de communs avec la souche $6 / 84$, dont les $6 \mathrm{~A}$ et $3 \mathrm{D}$,

Tableau VII. Analyse de variance, pour la vitesse d'extension des nécroses $\left(P_{\cdot \max }\right)$, des réponses des monosomiques contaminées par chaque souche de $S$. nodorum.

\begin{tabular}{|c|c|c|c|c|c|c|}
\hline \multirow[t]{2}{*}{ Variations } & \multirow[t]{2}{*}{ Somme des carrés } & \multirow[t]{2}{*}{ d.d.l. } & \multirow[t]{2}{*}{ C.M. } & \multirow[t]{2}{*}{$F$} & \multicolumn{2}{|c|}{ F. théorique } \\
\hline & & & & & $5 \%$ & $10 \%$ \\
\hline totale & 23055,44 & 79 & - & - & & \\
\hline souches & 2504,36 & 3 & 834,78 & 3,085 & 2,78 & 4,16 \\
\hline monosomiques & 5125,8 & 19 & 269,78 & 0,996 & 1,76 & 2,23 \\
\hline erreur & 15425,28 & 57 & 270,61 & - & & \\
\hline
\end{tabular}

Tableau VIII. Chromosomes de l'hôte qui, pour chaque souche de $S$. nodorum, augmentent ou réduisent $P$.max ou qui sont équivalents à la $F_{2}$ disomique.

Souches

Vitesse d'extension des nécroses foliaires $\left(P_{\max }\right)$

\begin{tabular}{|c|c|c|c|}
\hline 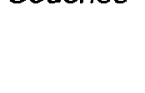 & $\begin{array}{l}\text { Réduction } \\
\text { (résistance) }\end{array}$ & Sans effet & $\begin{array}{l}\text { Augmentation } \\
\text { (sensibilité) }\end{array}$ \\
\hline $6 / 84$ & $\begin{array}{l}2 A-6 A-7 A \\
2 B-3 B-6 B \\
1 D-2 D-3 D\end{array}$ & $\begin{array}{l}1 A-3 A-4 A-5 A \\
1 B-4 B-7 B \\
5 D\end{array}$ & $4 D-6 D$ \\
\hline $6 / T 533-8$ & $\begin{array}{l}6 A \\
5 B \\
3 D\end{array}$ & $\begin{array}{l}1 A-5 A-7 A \\
1 B-3 B \\
1 D-2 D-4 D\end{array}$ & $\begin{array}{l}2 A-3 A-4 A \\
2 B-4 B-6 B-7 B \\
5 D-6 D\end{array}$ \\
\hline 6/T762 & $\begin{array}{l}3 A-5 A-6 A-7 A \\
4 B\end{array}$ & $\begin{array}{l}1 A-2 A \\
5 B-6 B-7 B \\
2 D-3 D\end{array}$ & $\begin{array}{l}4 A \\
1 B-2 B-3 B \\
1 D-4 D-5 D-6 D\end{array}$ \\
\hline $6 / T 832$ & $\begin{array}{l}3 A-4 A \\
2 B-5 B-6 B \\
3 D-4 D-6 D\end{array}$ & $\begin{array}{l}2 A-7 A \\
1 B-3 B-7 B \\
2 D-5 D\end{array}$ & $\begin{array}{l}1 A-5 A-6 A \\
4 B \\
1 D\end{array}$ \\
\hline
\end{tabular}


pour la résistance, et le $6 \mathrm{D}$ pour la sensibilité. Pour ces 2 mêmes souches, on relève des inversions de réponse oour les $2 \mathrm{~A}, 2 \mathrm{~B}, 5 \mathrm{~B}$ et $6 \mathrm{~B}$; celles-ci montrent que la part de l'agressivité prise en compte par $P_{\max }$ peut être dissociée en 2 éléments, les uns correspondant à des effets d'accroissement de $P_{\max }$, les autres à des effets de réduction. La pression de sélection exercée par le génome nucléaire de l'hôte s'exerce différemment suivant l'élément considéré de $P_{\max }$. La même remarque peut être formulée quant à l'action des cytoplasmes.

La diversification de la popualtion $6 / 84$, que révèle le passage sur le cytoplasme de $T$. timopheevi ou de $S$. cereale, est importante, puisque, par rapport à la souche 6/T533-8, seul le monosomique $2 \mathrm{D}$, qui est sans effet, donne une réponse de même sens aux 3 souches.

\section{Interactions monosomique $\mathrm{x}$ souche}

Elles ont été calculées globalement, par génome, et par groupe homéologue (Tableau IX) et sont significatives dans la quasi-totalité des cas étudiés.

Ces interactions sont globalement très élevées et, tout particulièrement, au niveau du génome $D$ pour la comparaison $6 / 84$ et $6 / 7832$. Ceci peut s'expliquer par la présence, chez le Triticale 832, à la fois du génome $\mathrm{R}$, non présent chez le blé, et du cytoplasme de seigle. Toutefois, cette hypothèse ne correspond pas au fait que c'est au niveau du génome $B$, présent chez le blé et les Triticale, que l'interaction entre les souches $6 / 84$ et $6 / 7533-8$ est la plus forte. Pour les groupes homéologues, on ne distingue pas statistiquement d'interactions pour le groupe I, quand on compare $6 / \mathrm{T} 533-8$ et $6 / \mathrm{T} 832$; pour les groupes $\mathrm{V}$ et $\mathrm{VI}$ et la comparaison $6 / \mathrm{T} 762$ et $6 / \mathrm{T} 832$; pour les groupes III et VI et la combinajson $6 / 84$ et $6 / T 762$; enfin pour la combinaison $6 / 84$ et $6 /$ T832 et les groupes I et III.

On peut identifier les chromosomes impliqués plus ou moins fortement dans ces effets différentiels (Tableau X). Aucun chromosome n'est sans effet différentiel pour l'ensemble des combinaisons étudiées. Comme pour la LPI, ce sont les souches $6 / 7762$ et $6 / 7832$ qui apparaissent les plus éloignées entre elles. Seuls 5 chromosomes de l'hôte sont sans effet différentiel, dont le 3A, qui réduit $P_{\max }$, et le 1D, qui au contraire l'augmente.

Pour les chromosomes qui donnent des effets différentiels, aucun n'est commun aux 4 souches comparées. Pour les souches qui ont subi la pression de sélection exercée par l'un des 3 cytoplasmes, seuls les $5 \mathrm{~A}, 6 \mathrm{~B}$ et $4 \mathrm{D}$ sont communs. Dans le cas des souches $6 / 7762$ et $6 / T 832$, qui ont subi les pressions de sélections exercées à la fois par les génomes nucléaire et cytoplasmique, 7 chromosomes avec effets différentiels sont communs avec la souche $6 / \mathrm{T} 533-8$; il s'agit des: 2A, 3A, 4A, 3B, 4B, 1D et 2D.

Ces résultats montrent que des pressions de sélection de nature différente ont été exercées sur la souche 6/84 et que celles qui combinent

Tableau IX. Valeur des $\mathrm{F}$ de l'interaction monosomique $\mathrm{x}$ souche, pour la vitesse d'extension des nécroses foliaires $\left(P_{\max }\right)$ calculée globalement, par génome et par groupe homéologue, pour les 6 comparaisons possibles.

Interactions

Comparaisons effectuées (1)

$\begin{array}{cccccc}6 / 84 & 6 / T 533-8 & 6 / T 533-8 & 6 / T 762 & 6 / 84 & 6 / 84 \\ \text { et } 6 / T 533-8 & \text { et } 6 / T 762 & \text { et } 6 / T 832 & \text { et } 6 / T 832 & \text { et } 6 / T 762 & \text { et } 6 / T 832\end{array}$

\begin{tabular}{|c|c|c|c|c|c|c|c|}
\hline Totale & & 16,86 & 7,38 & 9,52 & 63,83 & 19,55 & 205,31 \\
\hline \multirow{3}{*}{ par génome (1) } & $A$ & 8,32 & 19,23 & 69,82 & N.S. & 17 & 136,7 \\
\hline & B & 60,37 & 20 & 11,4 & 17,51 & 64,52 & 84,6 \\
\hline & $\mathrm{D}$ & 13,07 & 2,3 & 22,73 & 11,16 & 10,3 & 827,1 \\
\hline \multirow{6}{*}{$\begin{array}{l}\text { par groupe } \\
\text { homéologue (2) }\end{array}$} & I & 6,98 & 8,54 & N.S. & 205,87 & 31,32 & N.S. \\
\hline & II & 7,02 & 4,2 & 15,76 & 18,02 & 21,57 & 182,44 \\
\hline & III & 23,98 & 31,05 & 16,4 & 8,83 & N.S. & N.S. \\
\hline & IV & 156,74 & 14,33 & 3,88 & 45,52 & 9,45 & 38,7 \\
\hline & V & 8,62 & 11,06 & 46,5 & N.S. & 95,8 & 122,82 \\
\hline & VI & 4,24 & 3,51 & 130,62 & N.S. & N.S. & 209,12 \\
\hline
\end{tabular}

Le groupe homéologue VII n'est pas pris en compte du fait de l'absence du $7 \mathrm{D}$

(1) La valeur theorique de $F$ est 2,26 à $5 \%$ et 3,11 à $1 \%$.

(2) La valeur theorique de $F$ est 3,11 à $5 \%$ et 4,88 à $1 \%$.

N.S. : non significatif. 
Tableau X. Récapitulatif des chromosomes de l'hôte ayant, ou non, des effets différentiels sur la vitesse d'extension des nécroses foliaires $\left(P_{\max }\right)$ avec les souches du parasite.

\begin{tabular}{|c|c|c|c|}
\hline \multirow{3}{*}{$\begin{array}{l}\text { Comparaisons } \\
\text { effectuées }\end{array}$} & \multicolumn{3}{|c|}{ Chromosomes } \\
\hline & Avec effe & fférentiels & Sans effet \\
\hline & $\begin{array}{l}\text { Interactions élevées } \\
\text { (de résistant à sensible } \\
\text { et inversement) }\end{array}$ & $\begin{array}{l}\text { Interactions réduites } \\
\text { (de résistant ou sensible } \\
\text { à sans effet et inversement) }\end{array}$ & \\
\hline $\begin{array}{l}6 / 84 \text { et } 6 / T 533-8 \\
\text { effet génotype de T533-8 }\end{array}$ & $\begin{array}{l}2 \mathrm{~A} \\
2 \mathrm{~B}-5 \mathrm{~B}-6 \mathrm{~B}\end{array}$ & $\begin{array}{l}3 A-4 A-7 A \\
3 B-4 B-7 B \\
1 D-2 D-4 D-5 D\end{array}$ & $\begin{array}{l}{ }^{*} 1 A-{ }^{*} 5 A-6 A \\
{ }^{*} 1 B \\
3 D-6 D\end{array}$ \\
\hline $\begin{array}{l}6 / T 533-8 \text { et } 6 / T 762 \\
\text { effet cytoplasme } \\
T . \text { aestivum, T. timopheevi }\end{array}$ & $\begin{array}{l}3 A \\
4 B\end{array}$ & $\begin{array}{l}2 A-5 A-7 A \\
1 B-3 B-5 B-6 B-7 B \\
1 D-3 D-4 D\end{array}$ & $\begin{array}{l}{ }^{*} 1 A-4 A-6 A \\
2 B \\
{ }^{*} 2 D-5 D-6 D\end{array}$ \\
\hline $\begin{array}{l}6 / T 533-8 \text { et } 6 / 7832 \\
\text { effet cytoplasme } \\
\text { T. aestivum, Secale cereale }\end{array}$ & $\begin{array}{l}3 A-4 A-6 A \\
2 B-6 B \\
6 D\end{array}$ & $\begin{array}{l}1 A-2 A-5 A \\
7 B \\
1 D-4 D-5 D\end{array}$ & $\begin{array}{l}{ }^{*} 7 \mathrm{~A} \\
{ }^{*} 1 \mathrm{~B}-{ }^{*} 3 \mathrm{~B}-4 \mathrm{~B}-5 \mathrm{~B} \\
{ }^{*} 2 \mathrm{D}-3 \mathrm{D}\end{array}$ \\
\hline $\begin{array}{l}6 / \mathrm{T} 762 \text { et } 6 / \mathrm{T} 832 \\
\text { effet cytoplasme } \\
\text { T. timopheevi, Secale cereale }\end{array}$ & $\begin{array}{l}4 A-5 A-6 A \\
2 B-4 B \\
4 D-6 D\end{array}$ & $\begin{array}{l}1 A-7 A \\
1 B-3 B-5 B-6 B \\
3 D-5 D\end{array}$ & $\begin{array}{l}{ }^{*} 2 A-3 A \\
{ }^{*} 7 B \\
1 D-{ }^{*} 2 D\end{array}$ \\
\hline $\begin{array}{l}6 / 84 \text { et } 6 / T 762 \\
\text { effet génotype plus effet cytoplasme }\end{array}$ & $\begin{array}{l}2 B-3 B \\
1 D\end{array}$ & $\begin{array}{l}2 A-3 A-4 A-5 A \\
1 B-5 B-6 B \\
2 D-3 D-5 D\end{array}$ & $\begin{array}{l}{ }^{*} 1 \mathrm{~A}-6 \mathrm{~A}-7 \mathrm{~A} \\
4 \mathrm{~B}-{ }^{*} 7 \mathrm{~B} \\
4 \mathrm{D}-6 \mathrm{D}\end{array}$ \\
\hline $\begin{array}{l}6 / 84 \text { et } 6 / T 832 \\
\text { effet génotype plus effet cytoplasme }\end{array}$ & $\begin{array}{l}6 A \\
5 B \\
1 D-4 D-6 D\end{array}$ & $\begin{array}{l}1 A-2 A-3 A-4 A-5 A-7 A \\
3 B-4 B \\
2 D\end{array}$ & $\begin{array}{l}{ }^{*} 1 \mathrm{~B}-2 \mathrm{~B}-6 \mathrm{~B}-{ }^{*} 7 \mathrm{~B} \\
3 \mathrm{D}-{ }^{*} 5 \mathrm{D}\end{array}$ \\
\hline
\end{tabular}

* = Non différent des $\mathrm{F}_{2}$ disomiques pour les deux souches.

les génomes nucléaire et cytoplasmique donnent un résultat très différent de chacun de ces génomes pris isolément.

\section{Intensité des symptômes sur épis (IE)}

\section{Comparaisons globales}

L'analyse de variance montre que, tous monosomiques confondus, seules les souches se distinguent entre elles (Tableau XI).

Par I.E. croissante, elles se classent ainsi:

$6 / 84, \quad 6 / 7762, \quad 6 / 7533-8, \quad 6 / 7832$
(A)
$(\mathrm{AB})$
(B)
(B)

La souche $6 / T 832$ provoque la plus forte intensité de symptômes sur épis. Pour ce critère, les 3 cytoplasmes fournissent des souches d'agressivité comparable; c'est la pression de sélection exercée par le génome nucléaire de 6533-8 qui semble permettre l'émergence de souches différentes de la population originelle.

\section{Comparaison des monosomiques}

Le positionnement des monosomiques, par rapport aux disomiques, montre qu'il n'y a pas de monosomiques qui réduisent I.E. vis-à-vis des souches issues des diverses pressions de sélection exercées, sauf le 4B pour 6/T762 (Tableau XII).

De nombreux monosomiques (les 6A, 7A, 3B, $5 D$ et $6 D$ ) sont équivalents aux disomiques pour toutes les souches et les 2A, 5A, 3B et 7B sont équivalents aux disomiques pour les comparaisons entre les effets des cytoplasmes, à noyau constant. Dans ce dernier cas le 2D donne une réponse de sensibilité.

L'étude des interactions monosomique $x$ souche permet de préciser les différences ou les similitudes entre ces souches.

\section{Intéractions monosomique $x$ souche}

Globalement (Tableau XIII), elles ne sont significatives que pour la comparaison 6/T533-8 avec 
Tableau XI. Analyse de variance pour l'intensité des symptômes sur épis (IE) faite à partir de la note de chaque lignée exprimée en \% de la $F_{2}$ disomique.

\begin{tabular}{|c|c|c|c|c|c|c|}
\hline \multirow[t]{2}{*}{ Variations } & \multirow[t]{2}{*}{ Somme des carrés } & \multirow[t]{2}{*}{$d d l$} & \multirow[t]{2}{*}{$C M$} & \multirow[t]{2}{*}{$F$} & \multicolumn{2}{|c|}{$F$ théorique } \\
\hline & & & & & $5 \%$ & $10 \%$ \\
\hline totale & 177542,64 & 79 & - & - & & \\
\hline souches & 29126,64 & 3 & 9708,9 & 4,39 & 2,77 & 4,15 \\
\hline monosomiques & 22418,08 & 19 & 1179,9 & 0,533 & 1,76 & 2,23 \\
\hline erreur & 125947,62 & 57 & 2210,94 & - & & \\
\hline
\end{tabular}

Tableau XII. Chromosomes de I'hôte, qui, pour chaque souche de $S$. nodorum, augmentent ou réduisent l'intensité des symptômes sur épis (IE) ou qui sont équivalents à la $F_{2}$ disomique.

\begin{tabular}{|c|c|c|c|}
\hline \multirow{2}{*}{ Souches } & \multicolumn{3}{|c|}{ Intensité des symptômes sur épis (IE) } \\
\hline & $\begin{array}{l}\text { Réduction } \\
\text { (résistance) }\end{array}$ & Sans effet & $\begin{array}{c}\text { Allongement } \\
\text { (sensibilité) }\end{array}$ \\
\hline $6 / 84$ & $\begin{array}{l}2 A-4 A-5 A \\
1 B-3 B-6 B-7 B \\
2 D\end{array}$ & $\begin{array}{l}1 A-3 A-6 A-7 A \\
2 B-4 B-5 B \\
1 D-3 D-4 D-5 D-6 D\end{array}$ & \\
\hline 6/T533-8 & & $\begin{array}{l}1 A-2 A-5 A-6 A-7 A \\
2 B-3 B-6 B-7 B \\
3 D-4 D-5 D-6 D\end{array}$ & $\begin{array}{l}3 A-4 A \\
1 B-4 B-5 B \\
1 D-2 D\end{array}$ \\
\hline $6 / T 762$ & $4 \mathrm{~B}$ & $\begin{array}{l}2 A-3 A-4 A-5 A-6 A-7 A \\
1 B-3 B-5 B-7 B \\
1 D-3 D-5 D-6 D\end{array}$ & $\begin{array}{l}1 A \\
2 B-6 B \\
2 D-4 D\end{array}$ \\
\hline 6/T832 & & $\begin{array}{l}2 A-4 A-5 A-6 A-7 A \\
1 B-2 B-3 B-5 B-6 B-7 B \\
1 D-3 D-4 D-5 D-6 D\end{array}$ & $\begin{array}{l}1 A-3 A \\
4 B \\
2 D\end{array}$ \\
\hline
\end{tabular}

6/T762, qui met en jeu deux cytoplasmes, et pour la combinaison $6 / 84$ et $6 / T 762$, qui compare les effets combinés du noyau et du cytoplasme. Par génome, elles n'existent, sur le plan statistique, que dans 4 cas: pour les combinaisons 6/T533-8 avec $6 / T 762,6 / 84$ avec $6 / T 762$ pour le génome $A$, et les combinaisons $6 / \mathrm{T} 533-8$ avec $6 / \mathrm{T} 832$ et $6 / 84$ avec $6 /$ T762 pour le génome $D$.

Par groupe de chromosomes homéologues, des interactions ne sont détectées que dans 12 cas sur 36. Par exemple, elles n'existent pas pour le groupe IV pour toutes les comparaisons; elies n'existent, au niveau du groupe $\mathrm{VI}$, que pour la comparaison $6 / 7533-8$ et $6 / T 762$. Cette très grande différence dans les effets différentiels confirme que la réponse de l'épi est totalement indépendante de la réponse foliaire.

On peut identifier les chromosomes plus ou moins fortement impliqués dans ces effets différentiels (Tableau XIV). Globalement, le nombre de chromosomes aux effets non différentiels est élevé. II varie de 8 à 15; il semble que les souches $6 / 84$ et $6 / T 533-8$ sont les plus éloignées l'une de l'autre, puisqu'elles n'ont en commun que 8 monosomiques, qui sont à la fois sans effet différentiel, non différents de la réponse des disomiques et ayant 3 monosomiques avec des interactions élevées.

C'est pour les comparaisons $6 / 7533-8$ avec $6 / 7832$, et $6 / T 762$ avec $6 / 7832$ que les similitudes entre les souches sont les plus fortes.

Pour ce critère, on peut aussi relever que les pressions de sélection exercées par l'association dans un hôte de génomes nucléaire et cytoplasmique sont différentes de celles exercées par chacun de ces génomes pris isolément.

\section{Etudes comparées des critères d'expression de la maladie}

\section{$P_{\max }$ et LPI associés}

Quelles que soient les variations de la souche $6 / 84$, on retrouve la régression linéaire entre 
Tableau XIII. Valeur du F de l'interaction monosomique $x$ souche, pour l'intensité des symptômes sur épis (IE) calculé globalement, par génome et par groupe homéologue, pour les 6 combinaisons possibles.

\begin{tabular}{|c|c|c|c|c|c|c|c|}
\hline \multicolumn{8}{|c|}{ Comparaisons étudiées } \\
\hline Interactions & & 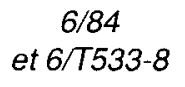 & $\begin{array}{l}6 / T 533-8 \\
\text { et } 6 / T 762\end{array}$ & $\begin{array}{l}6 / T 533-8 \\
\text { et } 6 / T 832\end{array}$ & $\begin{array}{c}6 / T 762 \\
\text { et } 6 / T 832\end{array}$ & $\begin{array}{c}6 / 84 \\
\text { et } 6 / T 762\end{array}$ & $\begin{array}{c}6 / 84 \\
\text { et } 6 / 7832\end{array}$ \\
\hline totale & & N.S. & 260,2 & N.S. & N.S. & 483,02 & N.S. \\
\hline par génome (1) & $\begin{array}{l}A \\
B \\
D\end{array}$ & $\begin{array}{l}\text { N.S. } \\
\text { N.S. } \\
\text { N.S. }\end{array}$ & $\begin{array}{r}255,11 \\
\text { N.S. } \\
\text { N.S. }\end{array}$ & $\begin{array}{l}\text { N.S. } \\
\text { N.S. } \\
79.2\end{array}$ & $\begin{array}{l}\text { N.S. } \\
\text { N.S. } \\
\text { N.S. }\end{array}$ & $\begin{array}{r}9,28 \\
\text { N.S. } \\
157,34\end{array}$ & $\begin{array}{l}\text { N.S. } \\
\text { N.S. } \\
\text { N.S. }\end{array}$ \\
\hline $\begin{array}{l}\text { par groupe } \\
\text { homéologue (2) }\end{array}$ & $\begin{array}{l}\text { I } \\
\text { II } \\
\text { III } \\
\text { IV } \\
\text { V } \\
\text { VI }\end{array}$ & $\begin{array}{r}\text { N.S. } \\
84,38 \\
13,65 \\
\text { N.S. } \\
23,43 \\
\text { N.S. }\end{array}$ & $\begin{array}{c}\text { N.S. } \\
\text { N.S. } \\
569 \\
\text { N.S. } \\
\text { N.S. } \\
9,53\end{array}$ & $\begin{array}{l}\text { N.S. } \\
\text { N.S. } \\
20.9 \\
\text { N.S. } \\
\text { N.S. } \\
\text { N.S. }\end{array}$ & $\begin{array}{c}\text { N.S. } \\
\text { N.S. } \\
18,11 \\
\text { N.S. } \\
\text { N.S. } \\
\text { N.S. }\end{array}$ & $\begin{array}{l}80,2 \\
14,47 \\
\text { N.S. } \\
\text { N.S. } \\
51,6 \\
\text { N.S. }\end{array}$ & $\begin{array}{r}\text { N.S. } \\
58,36 \\
54,87 \\
\text { N.S. } \\
\text { N.S. } \\
\text { N.S. }\end{array}$ \\
\hline
\end{tabular}

Le groupe homéologue VII n'est pas pris en compte du fait de l'absence du $7 \mathrm{D}$.

(1) La valeur théorique de $F$ est 2,26 à $5 \%$ et 3.11 à $1 \%$

(2) La valeur théorique de $F$ est 3,11 à $5 \%$ et 4,88 à $1 \%$.

N.S. : non significatif.

Tableau XIV. Récapitulatif des chromosomes de l'hôte ayant, ou non, des effets différentiels sur l'intensité des symptômes sur épis (IE) avec les souches de $S$. nodorum.

\begin{tabular}{|c|c|c|c|}
\hline \multirow{2}{*}{$\begin{array}{l}\text { Comparaisons } \\
\text { effectuées }\end{array}$} & \multicolumn{2}{|c|}{$\begin{array}{l}\text { Chromosomes } \\
\text { Avec effets différentiels }\end{array}$} & \multirow{2}{*}{$\begin{array}{l}\text { Sans effet } \\
\text { différentiels }\end{array}$} \\
\hline & $\begin{array}{l}\text { Interactions élevées } \\
\text { (de résistant à sensible } \\
\text { et inversement) }\end{array}$ & $\begin{array}{l}\text { Interactions réduites } \\
\text { (de résistant ou sensible } \\
\text { à sans effet et inversement) }\end{array}$ & \\
\hline $\begin{array}{l}6 / 84 \text { et } 6 / T 533-8 \\
\text { effet génotype de T533-8 }\end{array}$ & $\begin{array}{l}4 \mathrm{~A} \\
1 \mathrm{~B} \\
2 \mathrm{D}\end{array}$ & $\begin{array}{l}2 A-3 A-5 A \\
3 B-4 B-5 B-6 B-7 B \\
1 D\end{array}$ & $\begin{array}{l}{ }^{*} 1 A-{ }^{*} 6 A-{ }^{*} 7 A \\
{ }^{*} 2 B \\
{ }^{*} 3 D-{ }^{*} 4 D-{ }^{*} 5 D-{ }^{*} 6 D\end{array}$ \\
\hline $\begin{array}{l}6 / \text { T533-8 et } 6 / T 762 \\
\text { effet cytoplasme } \\
T . \text { aestivum, T. timopheevi }\end{array}$ & $4 \mathrm{~B}$ & $\begin{array}{l}1 A-3 A-4 A \\
1 B-3 B-5 B-6 B \\
1 D-4 D\end{array}$ & $\begin{array}{l}{ }^{*} 2 A-{ }^{\star} 5 A-{ }^{*} 6 A-{ }^{\star} 7 A \\
{ }^{\star} 3 B-{ }^{\star} 7 B \\
{ }^{\star} 2 D-{ }^{*} 3 D-{ }^{*} 5 D-{ }^{*} 6 D\end{array}$ \\
\hline $\begin{array}{l}6 / T 533-8 \text { et } 6 / \text { T832 } \\
\text { effet cytoplasme } \\
\text { T. aestivum, Secale cereale }\end{array}$ & & $\begin{array}{l}1 A-4 A \\
1 B-5 B \\
1 D\end{array}$ & $\begin{array}{l}{ }^{\star} 2 A-{ }^{*} 3 A-{ }^{*} 5 A-{ }^{\star} 6 A-{ }^{\star} 7 A \\
{ }^{\star} 2 B-{ }^{*} 3 B-{ }^{\star} 4 B-{ }^{*} 6 B-{ }^{\star} 7 B \\
{ }^{\star} 2 D-{ }^{*} 3 D-{ }^{*} 4 D-{ }^{*} 5 D-{ }^{*} 6 D\end{array}$ \\
\hline $\begin{array}{l}6 / \text { T762 et } 6 / \text { T832 } \\
\text { effet cytoplasme } \\
\text { T. timopheevi, Secale cereale }\end{array}$ & $4 B$ & $\begin{array}{l}3 A \\
2 B-6 B \\
4 D\end{array}$ & $\begin{array}{l}{ }^{\star} 1 A-{ }^{\star} 2 A-{ }^{\star} 4 A-{ }^{\star} 5 A-{ }^{\star} 6 A-{ }^{\star} 7 A \\
{ }^{*} 1 B-{ }^{*} 3 B-{ }^{\star} 5 B-{ }^{\star} 7 B \\
{ }^{*} 1 D-{ }^{\star} 2 D-{ }^{\star} 3 D-{ }^{\star} 5 D-{ }^{*} 6 D\end{array}$ \\
\hline $\begin{array}{l}6 / 84 \text { et } 6 / T 762 \\
\text { effet génotype plus effet cytoplasme }\end{array}$ & $\begin{array}{l}6 \mathrm{~B} \\
2 \mathrm{D}\end{array}$ & $\begin{array}{l}1 A-2 A-4 A-5 A \\
1 B-2 B-3 B-4 B-7 B \\
4 D\end{array}$ & $\begin{array}{l}{ }^{*} 3 A-{ }^{*} 6 A-{ }^{\star} 7 A \\
{ }^{*} 5 B \\
{ }^{*} 1 D-{ }^{*} 3 D-{ }^{*} 5 D-{ }^{*} 6 D\end{array}$ \\
\hline $\begin{array}{l}6 / 84 \text { et } 6 / T 832 \\
\text { effet génotype plus effet cytoplasme }\end{array}$ & $2 \mathrm{D}$ & $\begin{array}{l}1 A-2 A-3 A-4 A-5 A \\
1 B-3 B-4 B-6 B-7 B\end{array}$ & $\begin{array}{l}6 A-7 A \\
2 B-5 B \\
1 D-3 D-4 D-5 D-6 D\end{array}$ \\
\hline
\end{tabular}

* monosomiques non différents, pour les deux souches, des disomiques. 
$P_{\max }$ et la LPI (Tableau XV). Si la pente de la droite de régression n'est pas différente entre les souches $6 / 84,6 / T 533-8$ et $6 / \mathrm{T} 762$, elle est par contre significativement différente entre $6 / T 762$ et $6 / T 832$. Ceci peut traduire, d'une part, que les pressions de sélection exercées par les cytoplasmes de $T$. timopheevi et de $S$. cereale sont de nature différente et, d'autre part que les répercusions sur LPI et sur $P_{\max }$ ne sont pas identiques entre elles. En effet, on peut constater (Tableaux IV et VIII) que certains monosomiques (comme les $4 A, 6 D$ ), confrontés à la souche $6 / T$ 832 , donnent une réponse de sensibilité avec la LPI mais de résistance pour $P_{\max }$, alors que d'autres, comme les 6A, 4B, 1D, donnent une réponse de sensibilité à ces 2 critères. Cette observation confirme que les systèmes héréditaires qui gouvernent la LPI et $P_{\max }$, ne sont que pour partie liés entre eux.

II est aussi possible, en rapprochant les Tableau VI et $X$, de définir les relations entre ces 2 paramètres foliaires par le nombre de chromo-

Tableau XV. Caractéristiques des droites de régression entre $P_{\max }$ et LPI pour les 4 souches étudiées.

\begin{tabular}{llll}
\hline Souches & Pente & $\begin{array}{c}\text { Ordonnée } \\
\text { à l'origine }\end{array}$ & $\begin{array}{c}\text { Coefficient } \\
\text { de corrélation }\end{array}$ \\
\hline $6 / 84$ & 0,443 & $+48,06$ & $0,537 \mathrm{HS}$ \\
$6 / \mathrm{T} 533-8$ & 0,434 & $+53,77$ & $0,675 \mathrm{HS}$ \\
$6 / \mathrm{T} 762$ & 0,572 & $+43,21$ & $0,727 \mathrm{HS}$ \\
$6 / \mathrm{T} 832$ & 0,2656 & $+65,58$ & $0,491 \mathrm{~S}$ \\
\hline
\end{tabular}

$\mathrm{HS}=$ significatif au seuil de $1 \%$

$\mathrm{S}=$ significatif au seuil de $5 \%$. somes de l'hôte impliqués conjointement dans des effets différentiels ou non différentiels (Tableau XVI). Pour ces 2 paramètres, ce sont 8 à 16 chromosomes de l'hôte sur 20 qui sont impliqués dans des effets de même nature.

\section{LPI et IE associés}

Aucun des coefficients de régression entre ces 2 paramètres n'est significatif et le nombre de monosomiques intervenant dans des effets différentiels conjoints pour ces 2 critères est faible (Tableau XVI). Pour une souche donnée, les monosomiques donnant une réponse de sensibjlité ou de résistance sont au nombre de 4 , au maximum, pour la souche $6 / \mathrm{T} 533-8$ et de 2 , pour les autres souches (Tableau XVII).

\section{$P_{\max }$ et IE associés}

Là aussi, les coefficients de régression entre ces critères ne sont pas significatifs, quoique supérieurs à ceux calculés entre LPI et IE; le nombre de chromosomes de l'hôte donnant une même réponse de sensibilité ou de résistance à $P_{\max }$ et IE est compris entre 6 , pour la souche $6 / T$ 832, et 3 , pour la souche $6 / T 762$ (Tableau XVII). Toutefois, suivant les comparaisons effectuées, le nombre de monosomiques intervenant conjointement, dans des effets différentiels ou non pour ces 2 critères, est compris entre 14 et 8 (Tableau $\mathrm{XVI}$ ).

\section{LPI, $P_{\max }$ et IE associés}

Le nombre de chromosomes donnant une même réponse de sensibilité ou de résistance à 3 paramètres est très faible (Tableau XVII), 3 pour $6 / 7533-8$ et 1 pour $6 /$ T762. On constate aussi

Tableau XVI. Nombre de monosomiques impliqués, ou non, dans des effets différentiels, pour les critères de résistances pris 2 à 2 ou ensemble et pour les 6 comparaisons étudiées.

\begin{tabular}{|c|c|c|c|c|c|c|c|c|c|c|c|c|}
\hline \multirow{3}{*}{$\begin{array}{l}\text { Combinaisons } \\
\text { des critères } \\
\text { de résistance }\end{array}$} & \multicolumn{12}{|c|}{ Comparaisons étudiées } \\
\hline & \multicolumn{2}{|c|}{$\begin{array}{c}6 / 84 \\
\text { et } 6 / 7533-8\end{array}$} & \multicolumn{2}{|c|}{$\begin{array}{l}6 / T 533-8 \\
\text { et } 6 / T 762\end{array}$} & \multicolumn{2}{|c|}{$\begin{array}{l}6 / T 533-8 \\
\text { et } 6 / T 832\end{array}$} & \multicolumn{2}{|c|}{$\begin{array}{c}6 / T 762 \\
\text { et } 6 / T 832\end{array}$} & \multicolumn{2}{|c|}{$\begin{array}{c}6 / 84 \\
\text { et } 6 / T 762\end{array}$} & \multicolumn{2}{|c|}{$\begin{array}{c}6 / 84 \\
\text { et } 6 / 7832\end{array}$} \\
\hline & $I(1)$ & $N /(2)$ & 1 & NI & 1 & $N I$ & 1 & $N I$ & 1 & $N I$ & 1 & $N I$ \\
\hline LPl et $P_{\max }$ & 9 & 3 & 9 & 4 & 6 & 2 & 11 & 1 & 11 & 5 & 7 & 3 \\
\hline LPI et IE & 8 & 4 & 6 & 4 & 4 & 7 & 5 & 5 & 7 & 5 & 4 & 3 \\
\hline$P_{\max }$ et IE & 10 & 4 & 7 & 4 & 3 & 5 & 4 & 4 & 8 & 3 & 8 & 3 \\
\hline LPI et $P_{\max }$ et IE & 6 & 2 & 4 & 2 & 2 & 2 & 4 & 1 & 6 & 3 & 3 & 1 \\
\hline
\end{tabular}

(1) I = présence d'interaction

(2) NI = pas d'interaction 
Tableau XVII. Nombre de monosomiques donnant, pour chaque souche, une réponse de même sens (sensibilité ou résistance), avec critères de résistance combinés entre eux.

\section{Combinaisons \\ Souches étudiées}

des critères

de résistance

$6 / 84 \quad 6 / 7533-8 \quad 6 / T 762 \quad 6 / T 832$

$\begin{array}{lllll}\text { LPI et } P_{\max } & 4 & 9 & 6 & 6 \\ \text { LPI et IE } & 2 & 4 & 2 & 2 \\ P_{\max } \text { et IE } & 4 & 5 & 3 & 6 \\ \text { LPI et } P_{\max } \text { et IE } & 2 & 3 & 1 & 2\end{array}$

que le nombre de monosomiques impli-qués dans les interactions conjointes pour ces 3 critères est faible: de 2 à 6 (Tableau XVI).

\section{DISCUSSION ET CONCLUSION}

De nombreux auteurs (Robinson, 1969, 1980; Nelson, 1978; Rapilly 1988) assimilent, sur le plan des conséquences épidémiologiques, les termes de résistance partielle, de résistance générale, parfois de résistance au champ, à celui de résistance horizontale, tel que Van der Plank (1963) l'a proposé dans sa première approche des relations hôte-parasite. En 1978, cet auteur a précisé ses conceptions sur ce type de résistan$c e$, en énonçant les qualités que devaient présenter les relations hôte-parasite pour être qualifiées d'horizontales. Ce sont:

- Résistance horizontale ne veut pas dire polygénique; elle l'est souvent, mais ce n'est pas indispensable.

- Les variations de l'agressivité d'un parasite ne sont pas nécessairement corrélées avec les variations, chez l'hôte, des niveaux de résistance horizontale.

- Des relations de spécificité entre certains hôtes et certaines souches du parasite peuvent exister dans ce système.

- Seule l'absence de variation du rang de classement d'hôtes confrontés avec divers isolats d'un parasite permet de mettre en évidence ce type de résistance.

Nos résultats doivent donc être discutés par rapport à ces exigences, mais en considérant le type et la nature des pressions de sélection exercées, par les hôtes de passage, sur la population $6 / 84$ de $S$. nodorum; ils peuvent aussi être abordés par rapport au cas particulier: Triticum aestivum $x$ Septoria nodorum.
Le type de pressions exercées correspond, dans tous les cas, sur limbes foliaires, à une période de latence (de la contamination à la sporulation), suivie, sur épis, d'une période d'incubation (de la contamination à l'envahissement des tissus avec manifestation des symptômes de la maladie); ces 2 séquences (latence et incubation) étroitement liées (Rapilly et al., 1981) relèvent de la résistance partielle. Elles correspondent à 2 paramètres qu'il est nécessaire de prendre en compte pour juger d'une partie de la résistance générale des lignées de blé à ce parasite (Trottet \& Merien, 1982; Lancashire \& Jones, 1985; Stooksbury et al., 1987; Leonard, 1988).

Les pressions sélectives ont, pour origine, soit le génotype nucléaire, avec, sans doute, un effet particulier du génome $\mathrm{R}$ du Triticale qui remplace le génome $D$ du blé, soit le génome cytoplasmique ou encore les interactions nucléo-cytoplasmiques.

Les effets de ces pressions confirment que l'isolat $6 / 84$ de $S$. nodorum est une population composée d'une mosaïque de génotypes différents. Comme le suggèrent les travaux de Osbourn (1985), ces génotypes sont différents sans doute par leur noyau, mais rien ne permet de penser qu'ils ne le soient aussi par leur contenu cytoplasmique. En raison du faible nombre de cycles accomplis, un et demi, sur les hôtes de passage, il ne semble pas possible de penser que la mutagenèse ait joué un rôle dans les évolutions observées. Toutefois, pour Pyricularia oryzae, qui est aussi un parasite nécrotrophe, Chevaugeon \& Makounzi, (1981), Makounzi \& Chevaugeon (1981) ont montré que la recombinaison mitotique permettait de révéler, très rapidement, des variations de l'agressivité, qui s'accomplissaient lors du cycle végétatif du parasite. De même, Tolmsoff (1983) rappelle que l'hétéroploïdie peut expliquer une part de la variabilité des Fungi imperfecti. Nous ne pouvons, en l'état actuel de nos études, dire si ces 2 possibilités de variabilité existent chez $S$. nodorum.

II est aussi difficile de dire si ces variations sont stables dans le temps. Toutefois, nous avons constaté, d'une part, avec un isolement réalisé à partir de Triticale et, d'autre part, avec la souche 6/84 passée 1 fois sur limbes foliaires d'une lignée sœur du Triticale 533-8, puis 5 fois sur le blé «Etoile de Choisy", que nos n'avons pu réobtenir des souches dont le phénotype et le pouvoir pathogène étaient équivalents aux isolements de départ (Skajennikoff \& Rapilly, 1985).

Quelle que soit l'origine des variations constatées, il faut remarquer que, pour une souche donnée, les changements révélés ne s'exercent pas de la même manière sur la durée d'incubation (LPI), sur la vitesse d'extension des 
nécroses foliaires $\left(P_{\max }\right)$ ou sur l'intensité des symptômes sur épis (IE). Si, très souvent, des variations de même sens sont constatées pour LPI et $P_{\max }$, elles ne sont pas corrélées avec celles constatées pour IE, confirmant que résistance foliaire et résistance de l'épi relèvent de deux mécanismes indépendants.

Les 3 paramètres étudiés dépendent de plusieurs gènes. II apparaît, en particulier pour LPI et $P_{\max }$, que les pressions de sélection s'exercent sur le parasite de façons différentes sur la part qui diminue son agressivité (allongement de la $\mathrm{LPI}$, réduction de $\mathrm{P}_{\max }$ ) et sur la part qui accroît celle-ci.

Cette étude montre, en outre, que l'agressivité d'un parasite nécrotrophe peut être contre-sélectionnée par le génome nucléaire (comparaison 6/84 et 6/T533-8), par le génome cytoplasmique (comparaisons $6 / \mathrm{T} 533-8$ et $6 / \mathrm{T} 762,6 / \mathrm{T} 533-8$ et $6 /$ T832, $6 / T 762$ et $6 / T 832$ ), ou par l'interaction nucléocytoplasmique (comparaisons $6 / 84$ et $6 / T$ $762,6 / 84$ et $6 / 7832$ ). II n'y a pas d'additivité des effets «noyau" et "cytoplasme». Ces contre-sélections, mises en évidence par la réponse des chromosomes de l'hôte, ne sont pas identiques. Il est possible de définir un indice de similitude entre les souches pour chaque combinaison effectuée, et pour chaque critère de résistance pris en compte. Cet indice est compris entre 0 et 1 .

Ces indices (Tableau XVIII) confirment que les paramètres foliaires et celui de l'épi relèvent de 2 mécanismes différents. Ils montrent aussi que la LPI et $P_{\max }$ sont liés entre eux et que, sur feuille, les souches $6 /$ T762 et $6 /$ T832 sont plus éloignées l'une de l'autre; alors que sur épi, ce seraient les souches 6/84 et 6/T533-8 d'une part et d'autre part $6 / 84$ et $6 / 7762$. Les «forces" de

Tableau XVIII. Valeur de l'indice de similitude entre les souches combinées 2 par 2 et pour chaque critère de résistance.

Souches comparées

Indices de similitude (1)

$$
\text { LPI } P_{\max } \text { LPI et } P_{\max } \quad I E
$$

\begin{tabular}{lllll}
$6 / 84$ et $6 / T 533-8$ & 0,4 & 0,3 & 0,35 & 0,4 \\
$6 / T 533-8$ et $6 / T 762$ & 0,4 & 0,35 & 0,375 & 0,5 \\
$6 / T 533-8$ et $6 / T 832$ & 0,4 & 0,35 & 0,375 & 0,666 \\
$6 / T 762$ et $6 / T 832$ & 0,25 & 0,25 & 0,25 & 0,666 \\
$6 / 84$ et $T 762$ & 0,35 & 0,35 & 0,35 & 0,4 \\
$6 / 84$ et $6 / T 832$ & 0,5 & 0,3 & 0,4 & 0,45 \\
\hline
\end{tabular}

(1) $0=$ souches sans aucune similitude

1 = souches très similaires l'une de l'autre. sélection exercées par le noyau et par le cytoplasme sont du même ordre de grandeur. II faut rappeller que rares sont les cas connus de variation du pouvoir pathogène associée à un changement de cytoplasme de l'hôte. Le plus célèbre est le Helminthosporium maydis et le Phyllosticta maydis avec le cytoplasme "Texas" du maïs. Ce cas a été constaté, mais n'avait pas été prévu (Miller \& Koeppe, 1971). Magaih et al. (1968) signalent aussi pour le virus $X$ de la pomme de terre, sur piment, un cas d'hérédité maternelle. Enfin, pour les interactions nucléocytoplasmiques, on peut citer l'effet du cytoplasme de Aegylops ventricosa pour la résistance des blés au piétin verse (Doussinault et al., 1974). Notre étude apporte une démonstration des effets du cytoplasme hôte sur la variation de l'agressivité d'un parasite nécrotrophe: le Septoria nodorum.

Nos résultats montrent aussi que les variations de l'agressivité du parasite sont, pour partie, corrélées avec les niveaux de résistance horizontale des hôtes; en effet le Triticale 832 est celui qui, contaminé par la souche $6 / 84$, présente le plus haut niveau de résistance; viennent ensuite le Triticale 762 et enfin le 533-8 (Skajennikoff \& Rapilly, 1989). Ce classement ne correspond pas tout à fait à celui que permet de réaliser les indices de similitude calculés entre les souches. Enfin, les interactions indiquent que les souches acquièrent une certaine spécificité vis-àvis de leurs hôtes de passage provoquant, sur ceux-ci, des pertes de rendement supérieurs à celles obtenues avec la souche $6 / 84$ (Tableau $X \mid X)$. Une interrogation est donc posée sur la nature réelle de la pression de sélection qu'exerce l'hôte de passage sur le parasite.

Rappelons que pour la période de latence de la rouille brune de l'orge, Parlevliet (1979) avait déjà mis en évidence des interactions, signes de spécificité, entre les isolats du parasite et des variétés d'hôtes. Dans le même sens, soulignons le cas particulier du passage sur le Triticale 832, qui possède le cytoplasme de seigle. En effet, la souche 6/84 n'est pas pathogène sur le seigle, mais la souche $6 /$ T832, passée sur ce cytoplasme, le devient pour cet hôte, plus que la souche T/533-8, qui a subi la pression exercée par le génome nucléaire $R$ du seigle. Le cytoplasme seigle a donc conféré une certaine spécificité visà-vis de cet hôte inhabituel. II serait intéressant de vérifier si ces souches sont encore capables de réaliser des hétérocaryons (Newton \& Caten 1985), puisque Osbourn et al. (1986) ont montré que des isolats de blé et d'orge étaient incapables de réaliser entre eux des hétérocaryons.

Les variations de l'agressivité et ces spécificités nouvelles changent-elles les rangs de classement de variétés de blé vis-à-vis de souches de 
Tableau XIX. Pertes de rendement, en \% du poids de 1000 grains, du blé «Etoile de Choisy» ou des hôtes de passages, après 1 ou 4 passages de la souche 6/84 de $S$. nodorum sur ces hôtes.

\begin{tabular}{|c|c|c|c|c|c|c|c|}
\hline \multirow[b]{2}{*}{ Hôtes contaminés à l'épiaison } & \multicolumn{7}{|c|}{ Souches étudiées } \\
\hline & \multirow{2}{*}{$\begin{array}{c}6 / 84 \\
1 a\end{array}$} & \multicolumn{2}{|c|}{$6 / 7533-8$} & \multicolumn{2}{|c|}{$6 / T 762$} & \multicolumn{2}{|c|}{$6 / 7832$} \\
\hline & & $1 \mathrm{a}$ & $4 a$ & ta & $4^{a}$ & $1 \mathrm{a}$ & $4^{a}$ \\
\hline blé: Etoile de Choisy & 12 & 24,5 & 24,5 & 25,5 & 23 & 28 & 20,5 \\
\hline Triticale: T533-8 & 17,5 & 28 & 24,5 & - & - & - & - \\
\hline Triticale: T762 & 12 & - & - & 44 & 16,5 & - & - \\
\hline Triticale: T832 & 2,5 & - & - & - & - & 14,5 & 1 \\
\hline sigle: cv. Petkuss & 2,5 & 5 & - & - & - & 13 & 11,5 \\
\hline
\end{tabular}

ce parasite? En se référant à un travail antérieur, non publié, il nous est possible de donner une réponse. En effet, nous avons contaminé, avant gonflement ou à l'épiaison, 21 variétés de blé soit avec la souche 6 , très proche de $6 / 84$, soit avec la souche 42 , isolée à partir d'une lignée sœur de 533-8 (Rapilly et al., 1988b). Si le critère de classement des variétés, pour ces 2 souches, est l'évolution des épidémies, donnée qui intègre la résistance partielle foliaire, les coefficients de corrélation de rang sont hautement significatifs ( $r s=0,76$ pour les épidémies initiées au glonflement; 0,74 pour celles initiées à l'épiaison). Par contre, si les classements sont faits par rapport au poids relatif de 1000 grains (paramètre de tolérance), ces coefficients ne sont plus significatifs: ils valent 0,4 ou 0,19 , suivant l'époque des contaminations. L'une des caractéristiques de la résistance horizontale énoncées par Van der Plank est donc vérifiée, si l'on admet que la résistance partielle foliaire est un des éléments constitutifs de la résistance horizontale.

Les variations d'agressivité constatées peuvent être à l'origine de variations de la spécificité et donc éroder les niveaux de résistance. Mais il est difficile de savoir si cette érosion est stable dans le temps et donc présente un danger. En effet, nous avons constaté qu'après un quatrième passage sur Triticale, les pertes de rendement lues sur ces hôtes deviennent plus faibles (Tableau XIX) (Skajennikoff \& Rapilly, 1989).

Dans le cas particulier du couple Triticum aestivum, Septoria nodorum, cette étude permet de formuler des recommandations :

- Un isolat de Septoria nodorum est une population de génotypes. II est donc inutile, sauf si l'on veut rétablir une certaine variabilité, de vouloir contaminer les lignées à juger avec un mélange de souches.

- Tous les caractères pris en compte sont à hérédité polygénique. LPI et $P_{\max }$, les 2 critères de résistance foliaire, sont liés en partie, mais indé- pendants de l'expression de la maladie sur épi (IE). Résistance foliaire et résistance de l'épi sont à associer dans un programme de sélection.

- Le jugement de la spécificité relative, pour l'agressivité, d'isolats d'origines diverses doit être effectué sur feuilles et non sur épi.

L'agressivité d'un parasite peut être contresélectionnée par les génomes nucléaire et cytoplasmique des hôtes; l'utilisation de cytoplasmes autres que celui de $T$. aestivum peut donc présenter un risque parasitaire dans le cas particulier de $S$. nodorum.

Enfin, la résistance partielle, élément de la résistance générale, peut être erodée, elle doit être l'objet d'une prospective de gestion.

Septoria nodorum peut varier sans avoir recours à sa reproduction sexuée (Leptosphaera nodorum), mais les ascospores existent in natura, leur rôle, dans l'évolution des fréquences génotypiques de la population parasitaire, en fonction des pressions de sélection, est donc à élucider.

\section{RÉFÉRENCES}

Auriau P., Rapilly F. \& Cauderon Y. (1988) Analyse monosomique de la résistance à Septoria nodorum Berk. chez le blé tendre Triticum aestivum L. Agronomie 8, 71-77

Beye I. \& Lafay J.F. (1988) Verticilliose de la tomate: conséquences des interactions entre l'agressivité de l'agent pathogène et la résistance de l'hôte, au niveau des populations. Agronomie 8, 435-440

Cauderon Y., Cauderon A., Gay G. \& Roussel L.J. (1985) Alloplasmic lines and nucleocytoplasmic interactions on Triticale. In: Genetics and Breeding of Triticale. Eucarpia meeting, Clermont-Ferrand France, 1984 Ed. INRA. 181-184

Chevaugeon J. \& Makounzi J.A (1981) Variabilité de Puccinia oryzae Briosi Cov. en Afrique de l'Ouest. C.R. Symposium sur la résistance du riz à la pyriculariose. Montpellier, France IRAT GERDAT 154-166 
Doussinault G., Koller J., Touvin H. \& Dosba F. (1974) Utilisation des géniteurs UPM1 dans l'amélioration sanitaire du blé tendre. Ann. Amélior. Plantes 24, 215241

Flor H.H. (1955) Host parasite interaction in flux rust. Its genetic and other implications. Phytopathology 45, 680-685

Lancashire P.D. \& Jones G.D. (1985) Components of partial resistance to Septoria nodorum in winter wheat. Ann. Appl. Biol. 106, 541-535

Leonard K.J. (1988) Sporulation patterns to Septoria nodorum in wheat in relation to quantitative resistance. Z. Pflanzenbau Pflanzenschutz. 95, 337-346

Magaich B.B., Upadhya M.D., Pkakosh O. \& Singh S.J. (1968) Cytoplasmically determined symptoms of potato virus $X$ in cross between species of Capsicum. Nature 220, 1341-1342

Makounzi J.A. \& Chevaugeon J. (1981) Variabilité de l'agressivité de Pyricularia oryzae Briosi et Cov.. Effets de la mutation et de la recombinaison mitotique. C.R. Symp. sur la résistance du riz à la pyriculariose. Montpellier, France IRAT. GERDAT 192-204

Miller R.J. \& Koeppe D.E. (1971) Southern corn leaf susceptible and resistant mitochondria. Science 73 , 67-69

Nelson R.R. (1987) Genetics of horizontal resistance to plant disease. Ann. Rev. Phytopathol. 16, 359-378

Newton A.C. \& Caten C.E. (1985) Heterokaryosis and heterokaryon incompatibility in Septoria. In: Septoria of Cereals. (A.L. Sharen ed.). Dept of Agriculture ARS 12, pp. 13-15

Osbourn A.E. (1985) Host adaptation and variation in Septoria nodorum. Ph. D., Univ. of Birmingham, Dept 07 Genetics, U.K.

Osbourn A.E., Scott P.R. \& Caten C.E. (1986) The effects of host passaging on the adaptation of Septoria nodorum to wheat or barley. Plant Pathol. 35, 135145

Parlevliet J.E (1979) Components of resistance that reduce the rate of epidemic developement. Annu. Rev. Phytopathol. 17, 203-222

Rapilly F. (1984) Réflexions sur la résistance des plantes aux attaques parasitaires. Sél. Fr. 34, 5-18

Rapilly F. (1988) Partial resistance of wheat to Septoria nodorum Berk.: from the mathematical simulation to study of resistance chromosomes. Z. Pflanzenbau Pflanzenschutz 95, 419-427

Rapilly F., Auriau P., Laborie Y., Depatureaux C. \& Skajennikoff M. (1981) Résistance partielle du blé: Triticum aestivum L. à Septoria nodorum Berk. Agronomie 1, 771-782

Rapilly F., Auriau P., Cauderon Y., Richard H. \& Depatureaux C. (1988a) Réactions à la contamination par Leptosphaeria nodorum Müll. des feuilles, épis et grains de plantes $F_{2}$, issues du croissement entre une lignée de blé résistante et la série monosomique de Courtôt. Agronomie 8, 647-652

Rapilly F., Auriau P., Richard H. \& Depatureaux C. (1988b) Réactions d'un série monosomique de blé à la contamination des feuilles et des épis par deux souches de Septoria nodorum Berk. Agronomie 8, 5867

Robinson R.A. (1969) Disease resistance terminology. Rev. Appl. Mycol. 48, 593-606

Robinson R.A (1980) New concepts in breeding for disease resistance. Ann. Rev. Phytopathol. 18, 189210

Skajennikoff M. \& Rapilly F. (1985) Consequences of Triticale introduction on the parasitic fungi of wheat. In: Genetics Breeding of Triticale. Eurocarpia meeting, Clermont-Ferrand, France, Ed. INRA Paris pp 537549.

Skajennikoff M. \& Rapilly F. (1989) Variabilité du pouvoir pathogène chez Septoria nodorum Berk. Agronomie 9 (7)

Stooksbury D.E., Johnson J.W. \& Cunfer B.M. (1987) Incubation periods and latent period of wheat for resistance to Leptosphaeria nodorum. Plant. Dis. Rep. 71, 1109-1111

Tolmsoff W.J. (1983) Heteroploidy as a mechanism of variability among fungi. Ann. Rev. Phytopathol. 21, 317-340

Trottet M. \& Merien (1982) Analyse du comportement de 20 lignées de blé tendre vis-à-vis de Septoria nodorum. Agronomie 2, 727-734

Van der Plank J.E. (1963) Plant Disease: Epidemics and Control. Acad. Press

Van der Plank J.E. (1978) Genetic and Molecular Basis of Plant Pathogenesis. Springer Verlag 